\title{
Preface
}

\section{Up-to-Date on Orbital Trauma and Reconstruction}

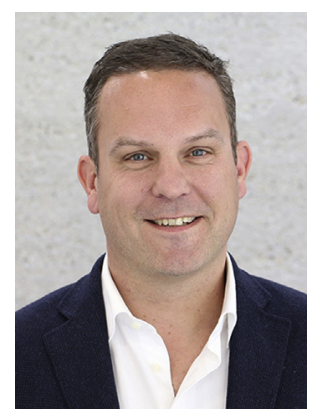

Leander Dubois, DDS, MD, DMD, PhD

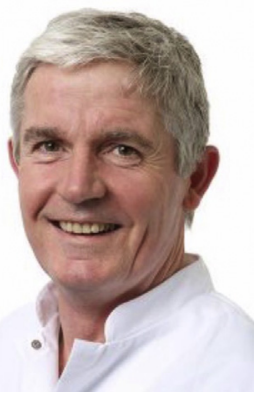

Alfred G. Becking, DDS, MD, DMD, PhD, FEBOMS
The orbit is a complex area with crucial anatomic structures packed into a small space. In orbital fractures not only the bone is involved but also the periorbit with vulnerable soft tissues. Orbital reconstruction is a delicate procedure with the protruding orbital soft tissues limiting the surgical overview. Technological developments like preoperative planning, navigation, additive manufacturing techniques, and intraoperative imaging have increased the predictability of the bony reconstruction in the recent years and are thought to have a favorable effect on the clinical outcome. So, anatomic knowledge and proper indications, combined with a good implemented clinical protocol can help to improve the quality of life for our patients. Above all, orbital fracture management has become a multidisciplinary matter.
This Atlas of the Oral and Maxillofacial Surgery Clinics of North America focuses on orbital fracture management for both primary and secondary trauma care. This issue begins with the anatomy of the orbit (Cornelius, Probst, Metzger, and Gooris), ocular injury and emergencies around the globe (Hötte and De Keizer), and the main controversies in orbital reconstructions regarding indications, timing, and biomaterials (Dubois, Dillon, Jansen, and Becking). In order to understand diplopia, the importance of orthoptic evaluation (Braaksma and Jellema) is underlined and how this can be implemented in an evidence-based clinical protocol (Gooris, Jansen, Bergsma, and Dubois). The primary fracture care (Holmes) and best implant for the right are further discussed. The fundamentals of $3 D$ virtual planning 
(Schreurs, Klop, and Maal) are explained and how this could be used for intraoperative feedback (Schreurs, Wilde, Schramm, and Gellrich). The complexity of secondary reconstructions is illustrated in solitary orbital fractures (Essig, Wagner, and Blumer) and in combination with NOE, ZMC, or panfacial fractures (Bell). The final article gives a scientific overview of which advanced technology could be used to improve the predictability of the reconstruction (Schreurs, Becking, Jansen, and Dubois).

We want to thank all the authors for their important contributions to this issue of Atlas of the Oral and Maxillofacial Surgery Clinics of North America. We especially wish to thank Nils Gellrich, Majeed Rana, and Harald Essig for introducing us to the field of navigation and Peerooz Saeed, Hinke Marijke Jellema, and Maarten Mourits for sharing their valuable insights in ophthalmology and orbital surgery.
Leander Dubois, DDS, MD, DMD, PhD Department of Oral and Maxillofacial Surgery Amsterdam University Medical Centers University of Amsterdam Academic Center of Dentistry Amsterdam Amsterdam, The Netherlands

Department of Oral and Maxillofacial Surgery St Antonius Hospital Utrecht/Nieuwegein/Woerden, The Netherlands

Alfred G. (Eddy) Becking, DDS, MD, DMD, PhD, FEBOMS Department of Oral and Maxillofacial Surgery Amsterdam University Medical Centers University of Amsterdam Academic Center of Dentistry Amsterdam Amsterdam, The Netherlands

Department of Oral and Maxillofacial Surgery MKA Kennemerland, The Netherlands

E-mail addresses:

I.dubois@amsterdamumc.nl (L. Dubois) ag.becking@amsterdamumc.nl (A.G. (Eddy) Becking) 